\title{
Development of Online Optical Fiber Communications Experiments using the Interactive ILabs Shared Architecture
}

\author{
Angelina Misso \\ Department of Electronics and \\ Telecommunications Eng. \\ University of Dar es Salaam, P.O. Box 33335, \\ Dar es Salaam Tanzania
}

\author{
Alfred Mwambela \\ Department of Electronics and \\ Telecommunications Eng \\ University of Dar es Salaam, P.O. Box 33335, \\ Dar es Salaam, Tanzania
}

\begin{abstract}
An intervention approach in introducing new online fiber optic communications labs in an operating environment where resources for establishing new conventional labs are limited is presented. The focus of this educational intervention is on the technological aspect required to develop and deploy the labs online. The developed labs demonstrated some of the distinctive features of fiber optic communications compared to the conventional electronics copper wire communications media. Telecommunications kits from Emona - FOTEx, National Instruments - ELVIS II hardware platform and MIT iLabs Shared Architecture remote lab framework were used to develop and deploy online fiber optic experiments. The developed labs have provided means for students to perform relatively new lab work anytime, anywhere. The sharing aspects extend their accessibility to other institutions with similar operating environment and limited technical knowhow on managing remote labs.
\end{abstract}

\section{General Terms}

Remote labs, Online Experiments, ILabs Shared Architecture.

\section{Keywords}

MIT ISA, Optic Fibers, FOTEx, ELVIS.

\section{INTRODUCTION}

In science and engineering courses offered in Universities, laboratory experiments play a major role in enabling the students to advance the theoretical knowledge obtained in class [1]. It is therefore necessary for any university offering such courses to have adequate and well equipped laboratories in order to effectively train students. However, limited financial resources, among others, for acquisition of new lab hardware remain a problem which in turn marginalizes the quality of the offered education. High cost of equipment like those required for fiber optics communications labs is one of the obstacles that universities encounter when it comes to provision of adequate lab equipment or introducing modern conventional labs. The problem is more compounded in developing countries where funding for higher education is a challenge, increased enrolment with unmatched increase in funding for related resources is common. Whereas they strive to enhance knowledge and support innovation, such operating environment constrains them in their aims at providing essential and modern skills and expertise required in the market.

Increased enrolments, which do not matches with the funding to increase associated resources, put a lot of strains on the existing infrastructure and human resources. For instance, between 2003 and 2006 the number of students registered at University of Dar es Salaam (UDSM) increased by $178 \%$. In 2006 alone, UDSM had around 17,000 enrolled undergraduate students, out of these; nearly 3,000 students were enrolled into science and engineering courses [2,3]. From these statistics, it is evident that the number of students is increasing every year. Unfortunately, this rapid increase in number of students is not at par with the available experimental facilities and funding to support related resources for such increased enrollment. Further, the existing sets of experiments are often old, adding or replacing the broken one becomes a challenge because they are no longer in production. Other restrictive factors include inadequate laboratory space and understaffing [4]. This prevailing challenging environment marginalizes the quality of the education offered. Where limited resources are available, most often it will be tied to maintaining the basic labs. Introducing advanced conventional labs such as optical fiber communications labs becomes a difficult proposition.

Increasing demand for high speed and bandwidth connectivity services has made fiber optics technology attractive for providing broadband services due to many advantages it offers such as high data rate and low attenuation loss over long distance. This has led to the development of global underwater optical fiber backbone connectivity with capacity of $20-505$ Gbps [5] and thereafter many national ICT broadband backbones were developed and linked to the global network [6]. These developments required universities to respond and embrace the technology by introducing fiber optics communication related courses. Two courses on fiber optics communication theory have been introduced for undergraduate second and third year students in order to help them understand the fundamental concepts on the field. However, introducing conventional labs for such courses was challenging because of the initial cost of buying equipment and maintenance involved.

Advances in computing industry have resulted in development of remote labs also referred to as online labs. Remote labs provide means for engineering and science students to perform experiments on physical laboratory equipment over the Internet in real-time. The idea behind is to develop real laboratory setups in a particular university, and these laboratories can be accessed by students in the same university and other subscribed universities from anywhere and anytime in the world through Internet access. As it turns out, remote labs offer an affordable solution to provision of labs in situation under discussed operating environment. Some of the advantages of remote labs are decreased fixed and variable expenditure on labs, scalability to reach more audience through sharing, and reduced startup costs of labs. 
This article describes the work carried out in the design and development of previously unavailable fiber optics labs for senior undergraduate courses at the University of Dar es Salaam. Online or web accessible labs which can be shared and accessed all the time from anywhere have been developed and deployed online using the interactive MIT ISA remote labs web services framework, NI ELVIS hardware platform, and the Emona FOTEx telecommunication kit. Five experiments unique to fiber optics communications have been implemented. The developed labs have provided means for students to perform relatively new lab work anytime, anywhere. The sharing aspects extend their accessibility to other institutions with similar operating environment and limited technical know-how on managing remote labs.

\section{ONLINE LABORATORIES}

Online laboratories provide means for engineering and science students to perform experiments on physical laboratory equipment over the Internet in real-time. Unlike traditional labs, remote labs do not require physical presence of the students in a laboratory. Online labs are convenient, flexible and offer easy management by the laboratory instructor. Through remote laboratories, students are able to select experiments and adjust experimental parameters online and get results. It also provides them with more time working on a particular experiment and hence being able to explore more. A significant advantage of iLabs is its ability to be shared among universities. Universities with insufficient resources can benefit since remote labs enable sharing of teaching approaches and lab designs [7].

A number of researches have been developed in support of development of online labs and they include iLabs [8] VISIR [9], WeCoLab [10] and LILA [11]. In this paper, iLabs has been selected and implemented for development of Optic Fiber Experiments. The other projects on online labs are based in Europe and Asia while iLabs through the Carnegie Project have invested in Africa. This influenced the decision to focus on iLabs as a platform for development of online optic fiber laboratories. In Africa, universities involved in the iLabs project are Makerere in Uganda, OAU in Nigeria and UDSM in Tanzania.

This paper is focused on the iLab Architecture. The iLab Shared Architecture (ISA) provides means to have Internet accessible laboratories [7]. The iLab project has invested in universities in Africa, including UDSM in Tanzania. They provide financial and technical support to member universities and this has facilitated the development of online experiments using the iLab Shared Architecture.

\section{ILAB SHARED ARTCHITECTURE}

Online ISA, which stands for iLab Shared Architecture was developed at Massachusetts Institute of Technology (MIT) in 1998 [8]. The iLab Shared Architecture provides a framework for lab development and deployment using web services. Basically, the iLabs Shared Architecture consists of three basic tiers which are the lab server, service broker and lab client which communicate using web services. The first tier is the Lab Client which provides an interface to the lab equipment and hardware using a Graphical User Interface (GUI) through which users can submit experiments and manipulate their results. The second tier is the Service Broker which deals with user authentication and registration, user authorization and credential management, experiment specification and result storage. The third tier comprises of the Lab Server, where the specified experiments are carried out on the actual laboratory hardware.

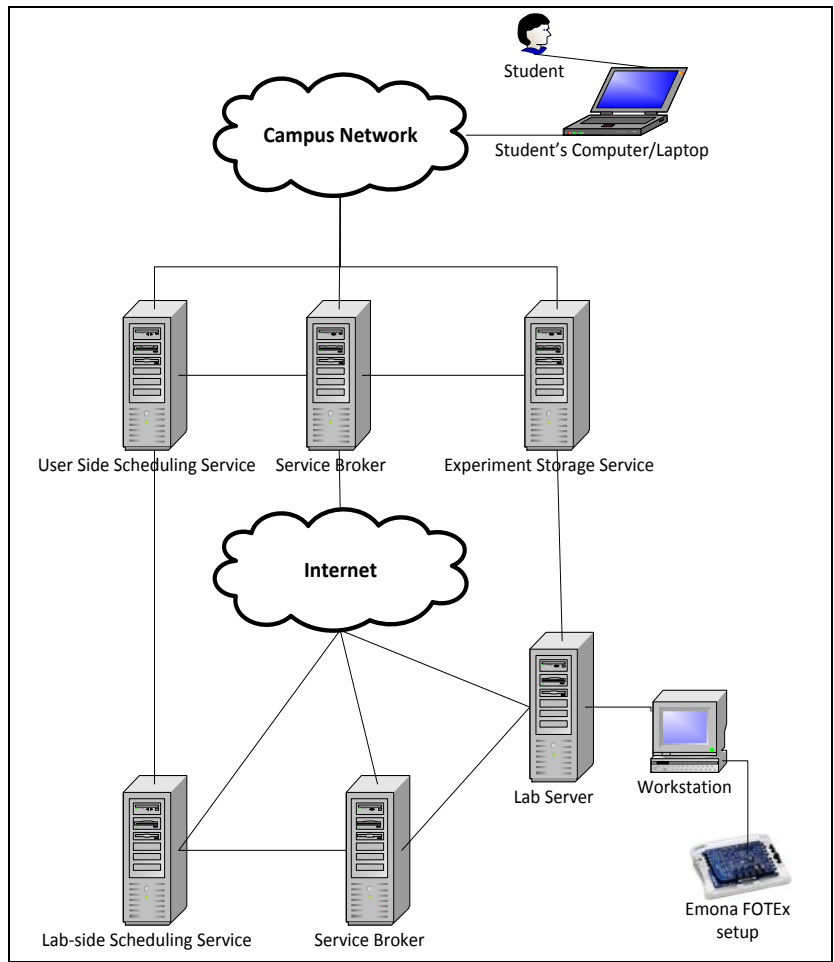

Figure 1: ISA interactive architecture

In terms of its operations, the ISA falls into three categories: interactive experiments, batched experiments and sensor experiments. For performing online laboratories, the batched and interactive ISA architectures were implemented at the time of doing this work. The Interactive ISA allows a user to vary parameters and observe the results in real time as they change which is different compared to batched ISA where parameters are defined before the experiment is queued and run as batched experiments involves queuing. The sensor ISA architecture is used in data collection [7].

Basically in an interactive iLab, lab clients and servers communicate directly; therefore the lab server and the lab client must be involved in the storage process. In addition to the three tier ISA components (Lab Client, Lab Server and Service broker), the interactive ISA includes three additional services which are: the Experiment Storage Service, Scheduling Services and Ticketing [8]. Scheduling is vital because the interactive experiments require that students have exclusive use of lab hardware for blocks of time. This research was focused on the Interactive ISA. Figure 1 shows the interactive iLab Architecture.

\section{FIBER OPTIC LABS DESIGN}

Fiber Optic communication experiments were selected at UDSM, because there were no laboratory facilities that could enable students to gain skills and knowledge on the basics of optic fiber communications to complement the knowledge and skills provided in the theoretical courses.

Fiber optics experiments from Emona, Fiber Optic Telecommunications Experiments (FOTEx) kit, from now on only referred to as FOTEx, is one of the many available fiber optics experiment kits based on the building blocks approach, which assist students in understanding complex theories implemented as real hardware. But one of the advantages it offers to this work is that the kit has been designed as an add-in board to NI ELVIS hardware platform. Many of the NI ELVIS functionalities have been integrated into the MIT ISA 
framework [8,12-14]; [15] and the platform has been extensively used in remote labs. These functionalities could be easily extended to FOTEx fiber optics kit. Further, others have used the kit for the same purpose.

The FOTEx kit consists of 14 communications building blocks, and was designed to support 13 experiments or labs, five of which are unique to fiber optics communications and are of interest to this work (Fiber optics transmission, Optical digital filtering, Fiber Optic Bidirectional communication, Wave division multiplexing and Optical losses). For online implementation purposes, the five labs were grouped into two sets of experiments; Experiment 1 consisting of fiber optics bi-directional communications, and Experiment 2 which consist of three experiments combined together to use single user interface.

The goal was to implement the two experiments which can enable students to gain the basics on optic fiber communication skills. During the experiment, students should be able to change parameters and observe changes as they occur, hence the need for interactivity. The target was to have students varying the following parameters: frequency, amplitude, signal type and sampling rate. At the output, the student will be able to observe the waveforms of the signals, and observe changes as the signal travels from source to destination. Students will have to schedule their sessions and therefore the laboratory equipment will be used by one student at a time. Consequently, the experiments were based on the Interactive iLabs Shared Architecture.

\section{SYSTEM DESIGN}

\subsection{System Requirements}

In order to deploy the experiments online, an analysis of the requirements was performed. This was vital in order to determine the hardware and software requirements. Before deploying the experiments online, it was necessary to have a circuit setup and a computer program to control the circuit remotely. First, it was required that the optic fiber experiments to be implemented on the Emona FOTEx as an add-on board on the NI ELVIS. Based on the two optic fiber experiments developed, the Emona FOTEx board and NI ELVIS II were the best hardware to be used. The Emona FOTEx and the NI ELVIS II were selected because they are interoperable. During the experiment session components from both equipment were used simultaneously. Furthermore, the equipment used i.e. Emona FOTEx and NI ELVIS II are compatible with ISA hence rendering them suitable for this research. Secondly, LabVIEW was used to program a VI that controlled the setup on the Emona FOTEx board. This VI was created in such a way that it could control both experiments. Finally, the interactive ISA platform was then developed and finally the experiments were launched online. Development of the iLab platform enables the interaction between the hardware-software interfaces, communication protocols and graphical user interfaces.

In this research four of these experiments were designed to form two major experiments. The fifth experiment: Fiber Optic Transmission was left out because it is automatically represented by the two deployed experiments. The first experiment (Experiment 1) is Fiber optic Bi-directional communication. The second experiment (Experiments 2) is Optical Fiber Communication system. The second batch of experiments consists of three individual experiments (Fiber Optic Signal filtering, WDM and losses experiments) that were combined to form one set of experiment (Experiment 2). These two experiments were implemented into the interactive ISA. Experiment two is more challenging as it combines three experiments into one. These experiments were chosen based on the fundamental optic fiber communication concepts offered in theory in order to help undergraduate engineering students in their second and third year of study in the University of Dar es Salaam to gain the basics of fiber optics. These concepts include general optical fiber communication, wavelength division multiplexing and optical losses.

Laboratory Virtual Instrument Engineering Workbench (Lab VIEW) is a graphical programming language that uses icons to create programs [16]. The VI developed, linked the computer to the experiments setup on the FOTEx. The VI consists of a number of blocks that enable the student to change parameters during the experiment. The parameters include: amplitude, frequency, offset, sampling values, signal type (analog or digital) and waveform (sinusoidal, square or triangular). The VI also comprised of the Function Generator, Oscilloscope and the DataSocket. The Function Generator was used to generate signals. The oscilloscope was used for observation of the waveforms. Data Socket allows easy data transfer over many different protocols (DSTP, OPC, LOOKOUT, HTTP, FTP, and local file access). The DataSocket simplifies live data sharing or exchange between different applications on one computer or between computers connected through a network.

\subsection{The Optic Fiber Experiments}

\subsubsection{Bi-Directional Communications}

One of the outstanding features of optical fiber is the fact that light travelling in one direction is unaffected by different light travelling in the reverse direction along the same fiber. As for electricity travelling through copper wires, when wires contain current flowing in different directions, the effect will be the currents cancelling each other out. This doesn't happen in optics because the signal carrier is light and light does not contain charged particles as electrons in electricity, it contains photons which are not charged particles. This is demonstrated in Figure 2.

This salient feature of supporting movement of signals in both directions in optical fibers without interference allows bidirectional communications. Optical couplers were used to load and unload signals at each transmitter and receiver. A coupler is a four port device used in optic communication that can support light to travel through fiber in two different directions. Couplers were used instead of circulators because they are cheap in comparison. Two signals of different frequencies that will travel along the fiber were used to demonstrate the experiment. 


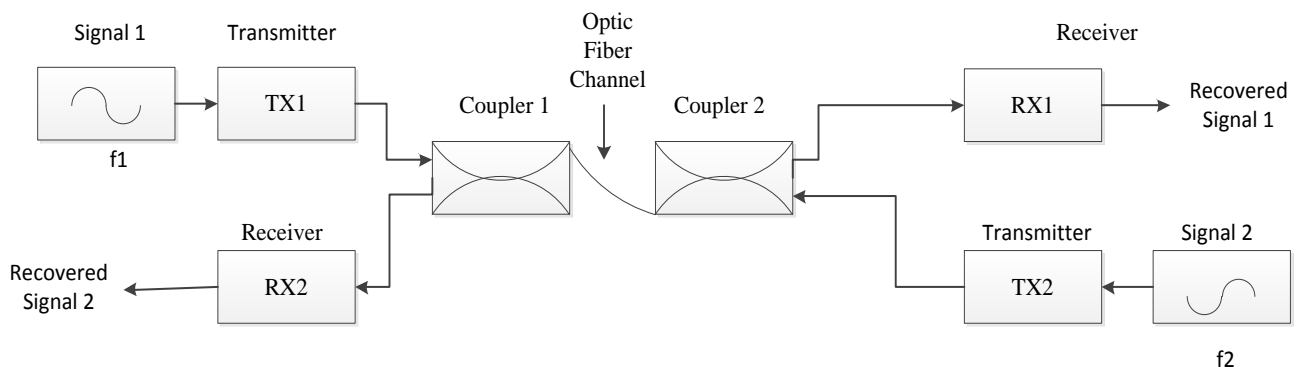

Figure 2: Bi-directional communications block diagram

\subsubsection{Signal Filtering, WDM and Losses System}

Figure 3 shows an optic fiber communication system with emphasis on the type of multiplexing used. The type of multiplexing commonly used in fiber is WDM. WDM stands for wavelength division multiplexing. One of the best features of optic fiber is its multiplexing ability. Multiplexing is the combining of two or more signals into a single wave from which the signals can be individually recovered. WDM works by spatially separating, or multiplexing, different wavelengths of light down a single optical fiber. WDM takes advantage of the fact that multiple colors, or frequencies, of light can be transmitted simultaneously down a single optical fiber. The data rate that each of the wavelengths supports depends on the type of light source. This experiment can also demonstrate optical losses, fiber optic filtering.

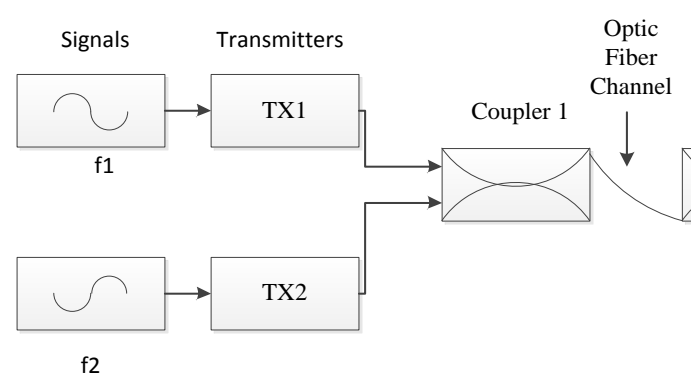

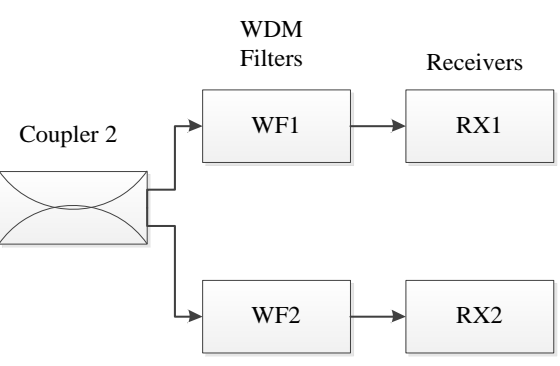

Figure 3: Optic fiber signal filtering, WDM and losses block diagram

In Optic fiber communication, multiple transmitted signals are then combined on the optical couplers and passed over a single fiber. At the receiving end where the signals are to be recovered, optic filtering occurs at the WDM filters. Optical filtering is when light of one particular wavelength to pass through it and blocks others. Optical filtering allows each specific transmitted wavelength to be recovered at their respective receivers. In the experiments performed in the Emona FOTEx, signals from different sources are carried via two different wavelengths, green and red light and are decoupled with the help of WDM filters (red and green).

Lastly, optical losses occur as the signal travels from the source to destination. In between it encounters several components that facilitate transmission but on the other hand, affect the signal quality. Sources of losses include: optical couplers and WDM filters.

\section{IMPLEMENTATION}

The implementation can be categorized into three parts: implementation of hardware components, software development and development of interaction between the ISA components. The first part in implementation was implementation of hardware components. In order to develop online optic fiber laboratory, it was required to develop a circuit on the FOTEx board and NI ELVIS. The circuit implementation is shown in figure 4. The Emona FOTEx and NI ELVIS II were selected because they are interoperable and they can be both controlled by a Virtual Instrument (VI) created on LabVIEW. Both boards were integrated into the
iLab platform hence making it possible for deployment of the fiber experiments on the iLab interactive platform. During the experiment session, communication blocks from both equipment (FOTEx and NI ELVIS) can be accessed simultaneously.

The second part was Software development. Programming for the experiments was done in Lab VIEW and a VI (virtual instrument) was created. This VI could control both experiments set up on the FOTEx board. The VI includes tabs that allow frequency, amplitude and signal type to be changed. It also includes an oscilloscope and table to display the results. Two signals could be viewed at a time on the VI front panel oscilloscope. The VI block diagram and front panel were presented in chapter three of this research.

Subsequently, an interaction between the iLab components and the experiment equipment was developed to allow communication between them. In an interactive iLab, all the components are interconnected. The Lab server is connected to the experiment equipment (NI ELVIS and FOTEx) and the Service broker performs authorization and authentication. To perform the experiments, the students required a Lab Client. The Lab Client used was Google Chrome which could load the front panel of the VI developed in Lab VIEW. The VI controlled the experiments that were set up on the FOTEx, an add-on board on the NI ELVIS. All this was done online because there was communication between the iLab components. 


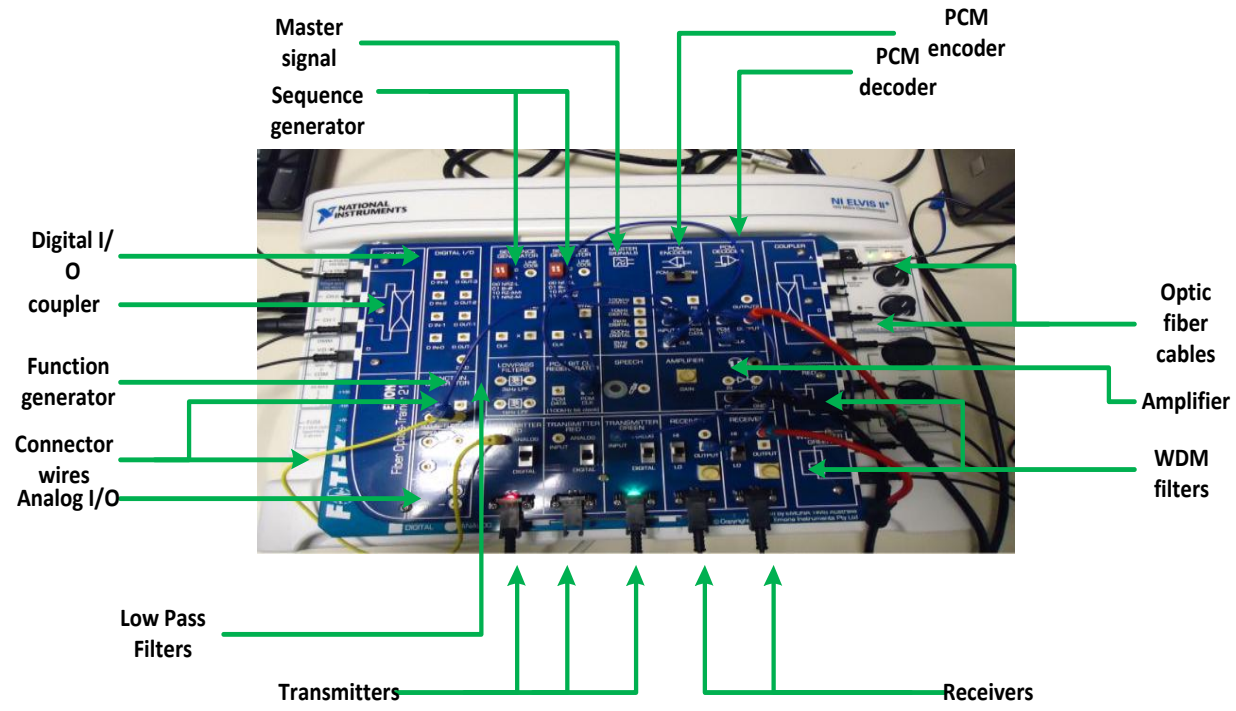

Figure 4: Experiment setup on the FOTEx board

\section{RESULTS AND DISCUSSION}

\subsection{Executing the Experiments}

In order to perform experiments in iLab, students are required to register on the Service Broker and request for membership in a group called "Communication group". The student is required to set reservation so as to obtain a slot to perform the experiments. The reservation includes selection for a date and time for performing the experiment. To do the experiment, click Launch Lab. Wait for the LabVIEW front panel to load and start working on the experiment. Figure 5 shows the user interface. This is what is displayed when students load the client. The results are displayed in graphical form. The student is able to adjust the amplitude and frequency of the signals, signal type, time base, sampling rate, among other parameters; and observe the changes as they occur on the screen.

The system was then tested to confirm the availability of the experiments online and if they work as desired. The implemented experiments are also tested to see if the circuits work as desired. The results from the two developed experiments are presented by using screen shots. Since the EMONA FOTEx can support two transmitted signals (it has red and green light for transmission of signals), two signals were transmitted from the source. Observations were made for:

- $\quad$ Signals with same frequencies and amplitudes.

- Signals with different frequencies and amplitudes

- Analog and digital signals

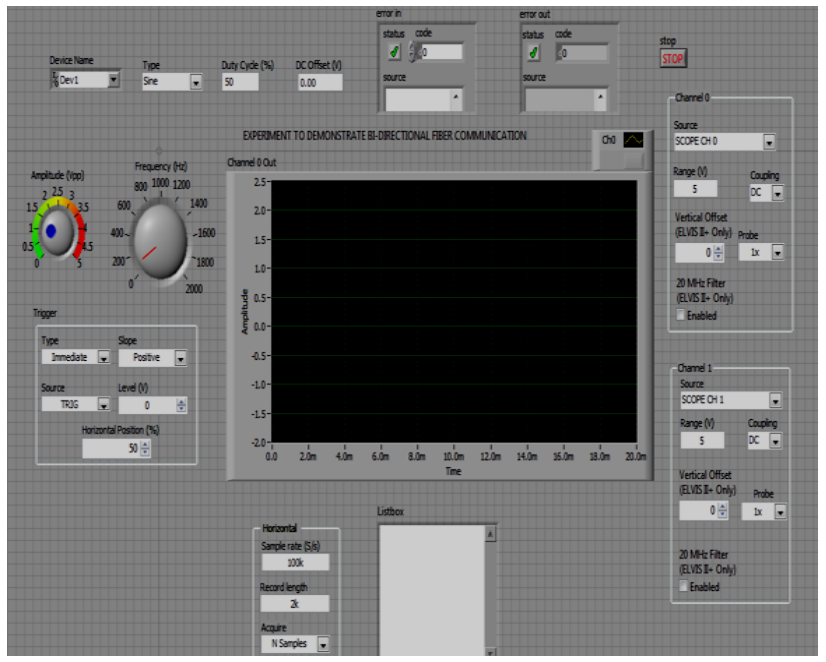

Figure 5: User interface

\subsubsection{Bi Directional Communications}

The students will be able to observe the effect of the signals on each other when transmitted in opposite directions by comparing input and output signals at each case.

Figure 6 shows two different signals were transmitted in opposite directions over the same fiber. The characteristics of the signals were varied. The first signal (controlled by function generator and carried by red light) has amplitude of $3 \mathrm{Vp}-\mathrm{p}$ and frequency of $200 \mathrm{~Hz}$ and it is indicated by yellow waveform on the oscilloscope display. The second signal (controlled by master signal block of the FOTEx board and carried by green light) has amplitude of $4 \mathrm{Vp}-\mathrm{p}$ and frequency of $2 \mathrm{kHz}$ and it is indicated by red waveform on the oscilloscope display. 


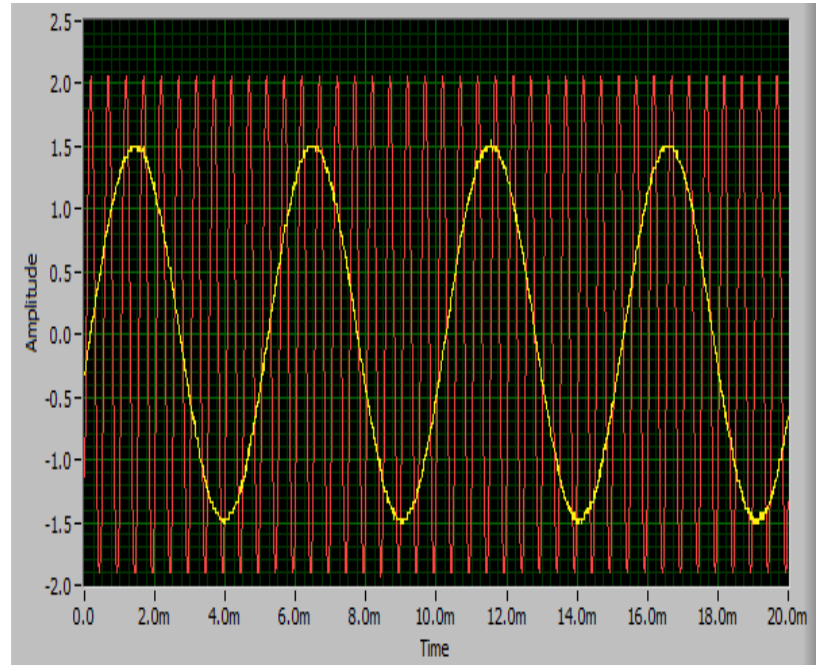

Figure 6: Bi-directional communications experimentinput signals

The results in Figure 7 show that the first signal is now superimposed over the second signal. This is a result of crosstalk caused by the couplers. Crosstalk arises because some of the signal on the port is reflected back due to the optical properties of the fiber fusion coupling. To solve the problem, the receiver output is passed through a low pass filter with cut off frequency above the highest frequency in the message.

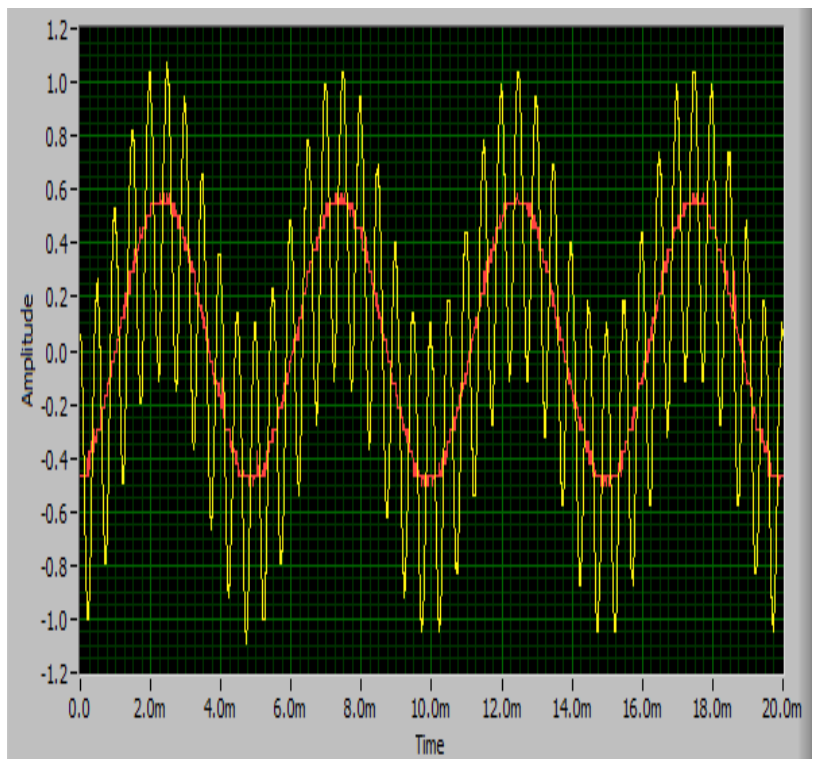

Figure 7: Bi-directional communication experimentoutput signals

\subsubsection{Signal Filtering, WDM and Losses System}

Two different signals were transmitted in the same directions over the same fiber. The characteristics of the signals were varied and observations were made. In this experiment, students will be able to observe the effect of filters, attenuation and WDM. The first signal (controlled by function generator and carried by red light) has amplitude of $2 \mathrm{Vp}-\mathrm{p}$ and frequency of $600 \mathrm{~Hz}$ and it is indicated by yellow waveform on the oscilloscope display. The second signal (controlled by the master signal block of the FOTEx board and carried by green light) has amplitude of $4 \mathrm{Vp}-\mathrm{p}$ and frequency of $2 \mathrm{kHz}$ and it is indicated by red waveform on the oscilloscope display as shown in Figure 8.
With optic fiber, each signal frequency is transmitted with a different wavelength. These wavelengths are later multiplexed together and carried over the channel to the receiver. At the receiver de-multiplexing is performed and the original signal can be recovered. So with different signal frequencies over the same channel, the results show high loss of fidelity of each of the signals as shown in Figure 9. As these pulses of light propagate through a fiber, they tend to lose their shape and spread out eventually overlapping each other causing intersymbol interference. This results into signal distortion. Analog signals are vulnerable to attenuation and noise. This is due to the fact that analog signals have continuous and varying amplitudes making it difficult to correctly decode the transmitted signal at the receiver. The waveforms have been shifted above the $0 \mathrm{~V}$ offset and are deformed with spikes occurring at different time instances. For example, the signal amplitude for the first signal is reduced from $2 \mathrm{Vp}-\mathrm{p}$ to $0.125 \mathrm{Vp}-\mathrm{p}$, making the loss to be $24 \mathrm{~dB}$ over the channel. The signal is reconstructed by passing it through a WDM filter and an amplifier to recover the original signal. This is shown in Figure 10.

N.B: The loss is calculated from the formula;

$$
\text { Attenuation }=20 \log _{10}\left(\frac{\text { output amplitude }}{\text { input amplitude }}\right)
$$

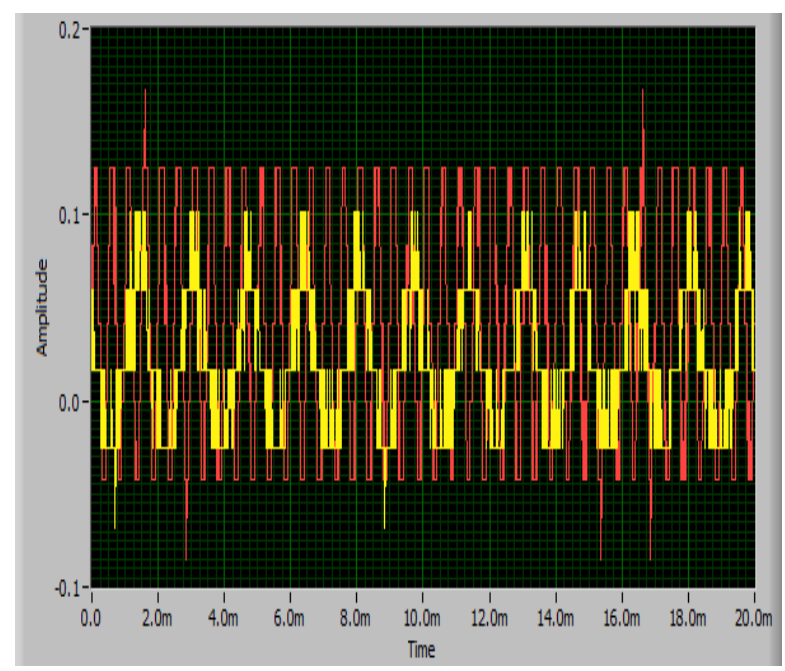

Figure 8: Optic fiber communication system experimentoutput signals without filtering.

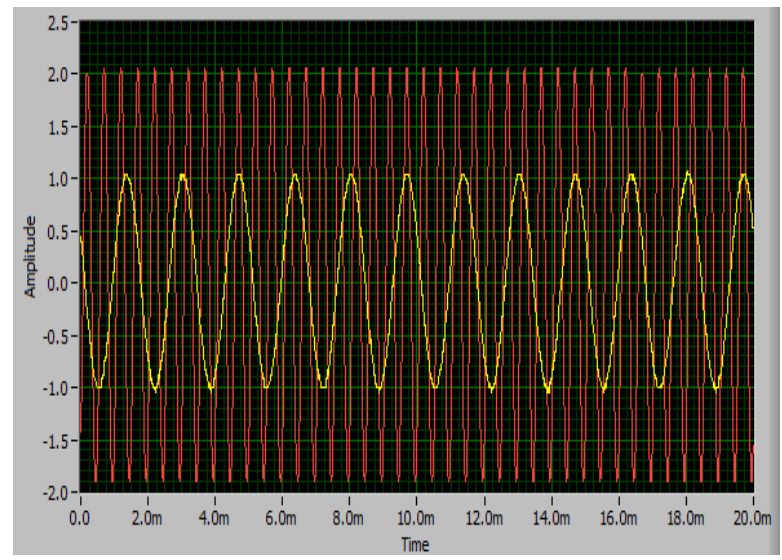

Figure 9: Optic fiber communication system experimentinput signals 


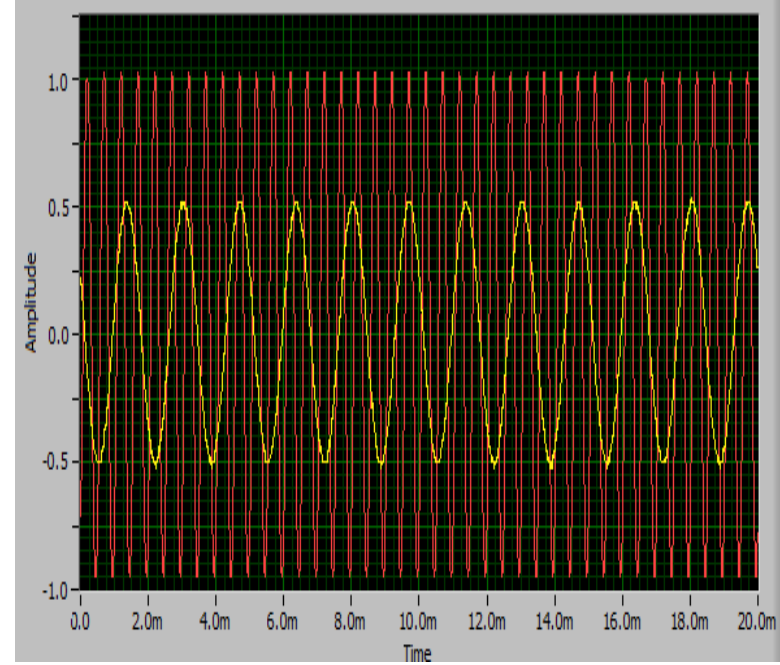

Figure 10: Optic fiber communication system experimentoutput signals after passing through WDM filters and passing through an amplifier to compensate for losses

\subsection{General System Performance}

\subsubsection{Bandwidth and Response Time}

The University of Dar es Salaam is connected by a fiber network of capacity of STM-1, which is a capacity of $155 \mathrm{Mbps}$; hence Internet speed was not a problem in implementing remote interactive optic fiber experiments. The time taken for the client user interfaces (web browser) to load the front panels of the Virtual Instruments for a given experiment was between 30 seconds to 45 seconds. The time between the registration processes, scheduling and launching of the experiment was a maximum of 4 minutes.

\subsubsection{Server Availability}

Occasionally the system may respond slowly for a period and then return to normal operation. These server slowdowns typically happen at peak traffic periods. These are issues of server hang-ups as a result of system overload which typically reveal as slowly loading pages.

\subsubsection{Number of Experiments that can be Remotely Performed on the FOTEx}

Two experiments were deployed on a single EMONA FOTEx board which was available. This made it difficult to switch between the two experiments remotely because this required human involvement in order to transfer of optic fiber cables between ports manually. One experiment could be performed remotely at a time until human intervention was utilized.

\section{CONCLUSION}

This research entailed the development and deployment of two online optic fiber experiments on the interactive iLab architecture. The Emona FOTEx can be used for development of general telecommunication experiments and optic fiber experiments. In this paper, the focus was on the optic fiber experiments where two experiments; optic fiber communication system and bi-directional communication were implemented successfully on the interactive iLabs Framework using the Emona FOTEx.

Future work on Emona FOTEx will be beneficial as it will intensify the use of Emona FOTEX by exploring on other experiments. The issue of switching between experiments can be solved by deployment of switching mechanism to allow remote transfer of control between the Emona components and hence support automatic shifting from one experiment to the next on the same board. The Analog input/output channels and digital input/output channels available on the NI ELVIS board could be used for implementation of the switch.

This signifies the successful completion on expanding the capabilities of the iLab shared Architecture to support Optic Fiber experiments. These experiments aim at enabling students to complement their theoretical knowledge and gain further knowledge in this interesting field of Optic Fiber Communication, by carrying out experiments remotely on the equipment located anywhere in the world as long as they have Internet connection.

\section{ACKNOWLEDGMENTS}

The authors would like to appreciate the contribution from the Carnegie Corporation through iLabs Africa project for the financial support offered through Grant No. BD03109.R04. Also the technical support from to the iLabs teams at Massachusetts Institute of Technology (MIT) in the United States of America and University of Dar es Salaam (UDSM) in Tanzania is highly appreciated.

\section{REFERENCES}

[1] Feisel L and Rosa A 2005 The role of the laboratory in undergraduate engineering education J. Eng. Educ. 94 121-30

[2] UDSM 2013 UDSM Facts and Figures

[3] SARUA 2011 UDSM Student Statistics

[4] Gikandi S 2006 ELVIS iLab: A Flexible Platform for Online Laboratory Experiments in Electrical Engineering (Master of Engineering Thesis, Massachusetts Institute of Technology.)

[5] Song S 2011 African Undersea Cables

[6] Pazi S, Chatwin C and Young R 2009 Performance of Tanzanian optical DWDM system Eur. J. Sci. Res. 36 606-26

[7] Harward V J, del Alamo J A, Lerman S R, Bailey P H, Carpenter J, DeLong K, Felknor C, Hardison J, Harrison B, Jabbour I, Long P D, Naamani L, Northridge J, Schulz M, Talavera D, Varadharajan C, Yehia K, Zbib R and Zych D 2008 The iLab Shared Architecture: A Web Services Infrastructure to Build Communities of Internet Accessible Laboratories Proc. IEEE 96 931-50

[8] Hardison J L, DeLong K, Bailey P H and Harward V J 2008 Deploying interactive remote labs using the iLab Shared Architecture 2008 38th Annual Frontiers in Education Conference (IEEE) pp S2A - 1-S2A - 6

[9] Gustavsson I, Zackrisson J, Håkansson L, Claesson I and Lagö T 2007 The VISIR project-an open source software initiative for distributed online laboratories Proceedings of the REV 2007 Conference, Porto, Portugal.

[10] Bochicchio M A and Longo A 2009 Hands-On Remote Labs: Collaborative Web Laboratories as a Case Study for IT Engineering Classes IEEE Trans. Learn. Technol. 2 320-30

[11] Richter T, Boehringer D and Jeschke S 2011 Lila: A european project on networked experiments Automation, Communication and Cybernetics in Science and Engineering 2009/2010 pp 307-17 
International Journal of Computer Applications (0975 - 8887)

Volume 131-No.11, December2015

[12] Mwambela A and Maiseli B 2012 Integrating the NI ELVIS Two-Terminal Current-Voltage Analyzer into the ISA Framework for Online Experiments Development Tanzania Eng. IET J. 12 84-90

[13] Sapula T and Haule D 2011 Implementation of Time and Frequency Response Analysis for Web-Based Laboratories J. Syst. Cybern. Informatics 9 1-6

[14] Sapula T and Haule D 2010 Development of Reliable Web-Based Laboratories for Developing Countries
World Acad. Sci. Eng. Technol. 4 259-65

[15] Jiwaji A, Hardison J, Ayodele K, Stevens TickodriTogboa S, Mwambela A, Harward V J, del Alamo J A, Harrison B and Gikandi S 2009 Collaborative development of remote electronics laboratories in the ELVIS ilab ASEE Annual Conference \& Exposition., Austin, TX, June 14-17 (American Society for Engineering Education)

[16] NI 2011 National Instruments: Lab VIEW 